\title{
AVALIAÇÃO TECNOLÓGICA DE PAINÉIS MDF DE MADEIRA DE Eucalyptus grandis CONFECCIONADOS EM LABORATÓRIO E EM LINHA DE PRODUÇÃO INDUSTRIAL
}

\author{
TECHNOLOGICAL EVALUATION OF Eucalyptus grandis WOOD MDF PANELS \\ MANUFACTURED IN LABORATORY AND INDUSTRIAL PRODUCTION LINE
}

\author{
Ugo Leandro Belini ${ }^{1}$ Mario Tomazello Filho ${ }^{2}$
}

\begin{abstract}
RESUMO
Chapas MDF de fibras da madeira de Eucalyptus grandis foram confeccionadas em laboratório e em linha de produção industrial e determinadas suas propriedades anatômicas, físicas e mecânicas. O desfibramento da madeira provocou o rompimento transversal da parede das fibras. Os painéis MDF obtidos em linha de produção apresentaram menores valores de inchamento e de absorção e melhores propriedades mecânicas para módulo de ruptura, módulo de elasticidade e resistência à tração superficial. Para os painéis MDF confeccionados em laboratório, verificou-se correlação estatisticamente significativa entre módulo de ruptura e de elasticidade, densidade média e resistência à tração perpendicular e inchamento e absorção. Para os painéis MDF confeccionados em linha de produção industrial, verificaram-se as mesmas tendências, sem significância estatística. A avaliação dos parâmetros de qualidade de painéis MDF de eucalipto confeccionados em laboratório e em linha de produção visa a calibrar as variáveis para a obtenção de chapas MDF em pequena escala e melhorar a precisão das análises de rotina de controle de qualidade bem como nas informações apresentadas em publicações científicas.
\end{abstract}

Palavras-chave: painéis MDF; fibras de Eucalyptus grandis; propriedades tecnológicas.

\begin{abstract}
MDF panels of Eucalyptus grandis wood fibers were made in either experimentally in a laboratory or on an industrial production line. In order to analyze the influence of the production condition, the anatomical, physical and mechanical properties of the panels were determined. The wood refining induced the transversal rupture of the transversal fiber wall. The MDF panels obtained from the industrial production line presented less swelling in thickness and absorption values and improved mechanical properties in the requirements of bending strength, module of elasticity and surface resistance. For laboratory MDF panels, it was possible to verify a statistically significant correlation between bending strength and module of elasticity, medium density and internal bond and swelling in thickness and absorption. This tendency was also true for the MDF panels obtained in real conditions of production, however without statistical significance. By comparing the quality properties of MDF panels produced in the laboratory with those obtained in real conditions of production, it was sought to standardize the established variables for obtaining panels on a small scale, as well to make possible the safe transfer and divulgation of information obtained in the laboratory.
\end{abstract}

Keywords: MDF panels; eucalypt fibers; technology properties.

1. Engenheiro Florestal, MSc., Doutorando pelo Programa de Pós-graduação em Recursos Florestais, Departamento de Ciências Florestais, Escola Superior de Agricultura Luiz de Queiroz, Universidade de São Paulo, Av. Pádua Dias, 11, Caixa Postal, 09, CEP 13418-900, Piracicaba (SP). ulbelini@esalq.usp.br

2. Engenheiro Florestal, Dr., Professor Titular do Departamento de Ciências Florestais, Escola Superior de Agricultura Luiz de Queiroz, Universidade de São Paulo, Av. Pádua Dias, 11, Caixa Postal, 09, CEP 13418-900, Piracicaba (SP).mtomazel@esalq.usp.br

Recebido para publicação em 23/03/2009 e aceito em 21/01/2010. 


\section{INTRODUÇÃO}

A grande aceitação dos painéis MDF pelo mercado brasileiro de indústrias de móveis e áreas relacionadas deve-se, dentre outras, à sua excelente trabalhabilidade no processo de usinagem e de acabamento superficial (BENADUCE, 1998; BNDES, 2002; IWAKIRI et al., 2005).

Nesse segmento da indústria de transformação da madeira do País, observa-se uma crescente tendência da participação da madeira de eucalipto na produção de painéis MDF (BELINI, 2007) produzidos, basicamente, pela mistura dos elementos fibrosos com adesivo sintético seguida de prensagem sob ação de calor, de acordo com a norma ABNT NBR 15316-1 (2006).

A confecção e a determinação das propriedades físico-mecânicas de painéis MDF de madeira de eucalipto, em condições de laboratório, têm sido realizadas por diversos autores (KRZYSIK et al., 2001; GORRINI et al., 2004; CAMPOS, 2005). No entanto, é fundamental comparar as propriedades tecnológicas dos painéis MDF obtidos nessas condições com as de linha de produção, visando a determinar quais propriedades podem ser significativamente semelhantes e/ou diferentes.

A confiabilidade dos resultados das análises dos painéis MDF confeccionados em laboratório é importante, não somente na rotina de controle de qualidade na linha de produção industrial, bem como no desenvolvimento de novos produtos e metodologias e da elaboração de artigos científicos com resultados confiáveis, direcionados para o setor florestal-industrial e para a comunidade científica.

Pelo exposto, no presente trabalho foram confeccionados painéis MDF de madeira de Eucalyptus grandis em laboratório e em linha de produção industrial com objetivo de comparar suas propriedades anatômico-físico-mecânicas.

\section{MATERIAL E MÉTODOS}

\section{Espécie e coleta das amostras de madeira após desfibramento}

Troncos de árvores de Eucalyptus grandis de 6,9 anos foram transformados em cavacos de madeira em picador industrial e, em seguida, desfibrados nas condições de (i) tempo de pré-aquecimento: 4 minutos; (ii) energia específica: $100 \mathrm{kwh} / \mathrm{t}$; (iii) pressão de digestão: 8,0 bar; (iv) pressão caixa de discos: 8,2 bar, conforme Belini et al. (2008a). Amostras de madeira desfibrada foram coletadas na esteira de formação do colchão, de forma aleatória, no sentido do fluxo de produção, após a encolagem com (i) resina uréia formaldeído 11,0\% (sólidos resina/fibra seca), (ii) emulsão de parafina $0,37 \%$ (sólidos emulsão/fibra seca) e (iii) catalisador sulfato de amônia $0,8 \%$ (sólidos de catalisador/resina), secagem e classificação, de acordo com Belini et al. (2008b)

\section{Caracterização anatômica dos componentes celulares da madeira}

Foram coletados $10 \mathrm{~g}$ de material desfibrado da madeira de eucalipto para a classificação morfológica dos componentes celulares via úmido em equipamento Bauer McNett modelo 203C (TAPPI, 1975). As amostras dos componentes celulares do lenho retidos nas peneiras 20-35-65150 mesh foram coletadas, armazenadas a $37^{\circ} \mathrm{C} \mathrm{em}$ frascos plásticos com água e formol $(80 \mathrm{ml}+1 \mathrm{ml})$. As células do lenho com corante safranina $(50 \%$ em solução alcoólica) e glicerina foram transferidas para lâminas de vidro e examinadas sob microscopia de luz (JOHANSEN, 1940). Foram coletadas cem imagens das células/lâmina e mensuradas as suas dimensões (comprimento, largura, espessura da parede, diâmetro do lume) de acordo com a IAWA Committee, (1989). Para o comprimento das fibras foram mensuradas 396 fibras (99 fibras/peneira/ repetição), para a sua largura, espessura da parede e diâmetro do lume, 300 fibras (75 fibras/peneira/ repetição) (ECKBLAD, 1991).

\section{Confecção dos painéis MDF em condições de laboratório}

Amostras de material fibroso da madeira de Eucalyptus grandis foram coletadas na esteira de formação do colchão de fibras, em linha de produção industrial de painéis MDF, separandoas em seis subamostras de $2,0 \mathrm{~kg}$ de um total de $12 \mathrm{~kg}$. Em seguida, a massa de fibras de cada subamostra foi distribuída manualmente em caixa formadora de colchão (390 x 390 × 300 mm) e préprensada para a retirada do ar e para proporcionar a compactação inicial. Na sequência, o colchão de fibras foi prensado em prensa laboratorial marca Siempelkamp, a $195^{\circ} \mathrm{C}$, no ciclo de prensagem de $10 \mathrm{~s}\left(0\right.$ a $\left.100 \mathrm{~N} / \mathrm{cm}^{2}\right), 5 \mathrm{~s}\left(100 \mathrm{~N} / \mathrm{cm}^{2}\right), 20 \mathrm{~s}(100$ a 20 $\left.\mathrm{N} / \mathrm{cm}^{2}\right), 15 \mathrm{~s}\left(20\right.$ a $\left.10 \mathrm{~N} / \mathrm{cm}^{2}\right), 65 \mathrm{~s}\left(10 \mathrm{~N} / \mathrm{cm}^{2}\right), 50 \mathrm{~s}$ $\left(30 \mathrm{~N} / \mathrm{cm}^{2}\right), 5 \mathrm{~s}\left(0 \mathrm{~N} / \mathrm{cm}^{2}\right)$. As seis chapas de painéis

Ci. Fl., v. 20, n. 3, jul.-set., 2010 
MDF confeccionadas com densidade nominal de 700 $\mathrm{kg} / \mathrm{m}^{3}$ foram climatizadas à temperatura ambiente e refiladas (18,9 x 380 × $380 \mathrm{~mm})$. O fluxograma do processo produtivo de painéis MDF em condições de laboratório é apresentado na Figura 1A.

\section{Confecção de painéis MDF em linha de produção industrial}

Em linha de produção industrial, as fibras com a mistura de resina, emulsão e de catalisador foram prensadas em prensa contínua marca Siempelkamp modelo ContiRoll, aplicando-se o mesmo ciclo de prensagem utilizado em escala laboratorial, exceto pelo gradiente de temperatura dos pratos da prensa $\left(205^{\circ} \mathrm{C}\right.$, início; $175^{\circ} \mathrm{C}$, término), obtendo-se painéis MDF (18,9 x 370 × $370 \mathrm{~mm})$ na densidade nominal de $700 \mathrm{~kg} / \mathrm{m}^{3}$. O fluxograma do processo produtivo de painéis MDF em linha de produção industrial é apresentado na Figura 1B.
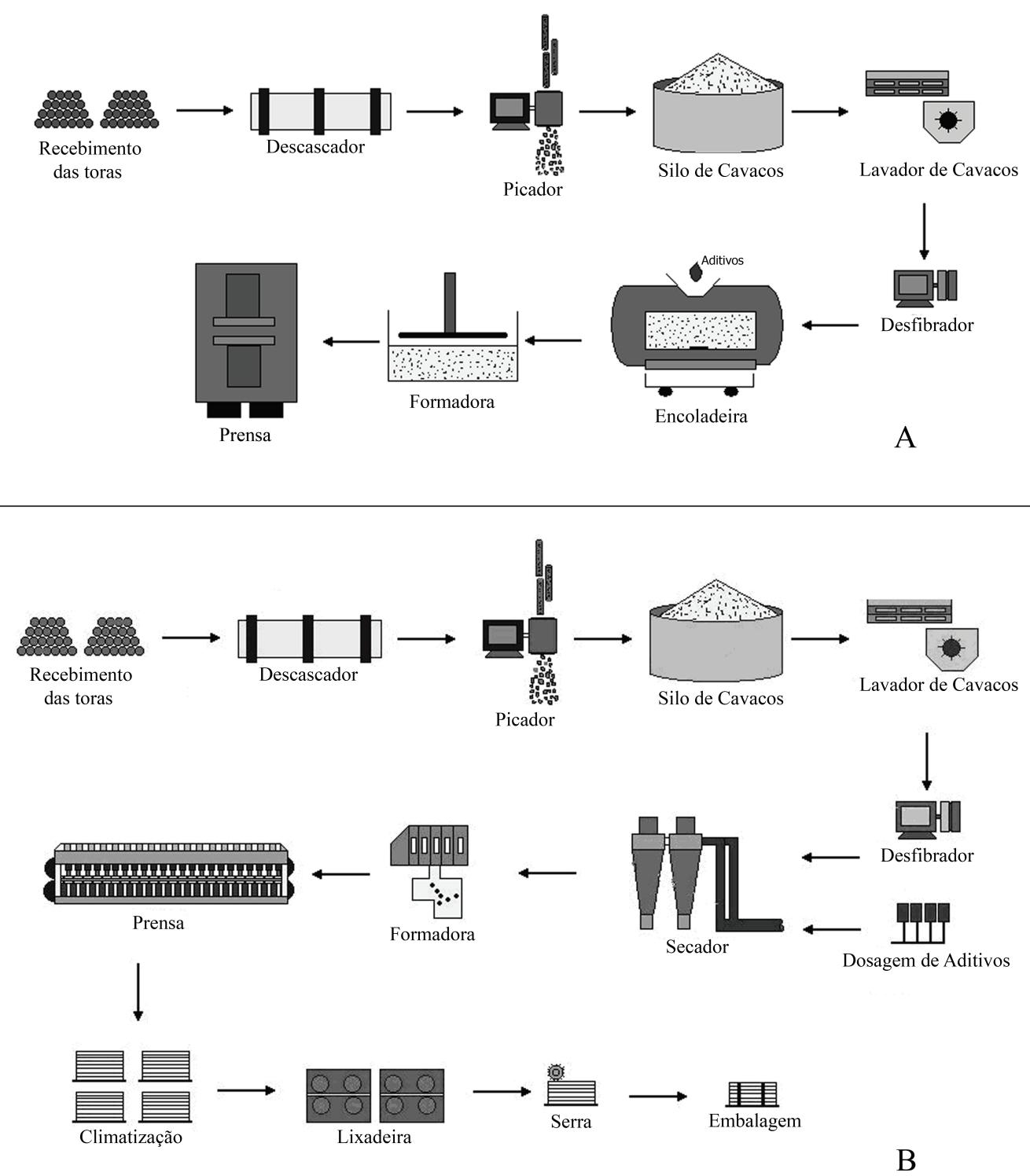

FIGURA 1: Fluxograma da produção de painéis MDF (A) em condições de laboratório e (B) em linha de produção industrial.

FIGURE 1: Flowchart for MDF panels production. (A) in laboratory and (B) in industrial line. 
Determinação das propriedades físico-mecânicas dos painéis MDF

As propriedades físicas (densidade $\mathrm{e}$ inchamento/absorção após 24 h) e mecânicas (resistências às trações perpendicular e superficial, arrancamento de parafuso, módulo de rupturaMOR e módulo de elasticidade-MOE) dos corpos de prova foram determinadas em equipamento de ensaio universal em atendimento à norma $\mathrm{ABNT}$ NBR 15316-3 (2006). Para as condições de laboratório e de linha de produção, foram analisadas 24 amostras (inchamento e absorção, resistência à tração perpendicular ao plano e resistência superficial) e 16 amostras (MOR, MOE e resistência ao arrancamento de parafuso) em delineamento estatístico inteiramente aleatorizado.

\section{RESULTADOS E DISCUSSÃO}

Caracterização anatômica dos componentes celulares da madeira após desfibramento

A avaliação das dimensões das fibras retidas nas diferentes peneiras de classificação indica uma diminuição do seu comprimento na peneira 150 mesh, pelo rompimento transversal da parede das fibras na fase de desfibramento dos cavacos de madeira (Tabela 1). A literatura relata para a madeira de diferentes espécies de eucalipto o comprimento médio das fibras em $1 \mathrm{~mm}$ (SILVA et al., 2005), incluindo o Eucalyptus grandis (TOMAZELLO F ${ }^{\mathrm{O}}, 1985 \mathrm{ab}$; ANDRADE et al., 1994), sendo superior ao das fibras mensuradas nas peneiras mais restritivas.

No lenho de árvores de Eucalyptus grandis, aos 7 anos, Rocha et al. (2004) mensuraram fibras com $942,19,9,12,3$ e $3,8 \mu \mathrm{m}$ para o comprimento, largura, diâmetro do lume e espessura da parede respectivamente. A espessura da parede das fibras retidas nas diferentes peneiras é superior à obtida por Rocha et al. (2004), possivelmente como resultado da deposição do adesivo uréiaformaldeído na etapa de encolagem. Para largura e diâmetro do lume das fibras os valores obtidos são similares aos da literatura (ANDRADE et al., 1994; ROCHA et al., 2004; TOMAZELLO Fo, 1985b). Como a madeira de eucalipto desfibrada apresentou parâmetros anatômicos similares, notadamente para as dimensões das fibras, e foram utilizadas para a confecção dos painéis em laboratório e linha de produção, infere-se que as diferenças observadas nas propriedades físicomecânicas dos painéis devem-se a sobretudo às características de prensagem dos equipamentos de laboratório e linha de produção.

TABELA 1: Dimensões das fibras de madeira de eucalipto após desfibramento.

TABLE 1: Dimensions of eucalypt fibers after refining.

\begin{tabular}{lcccc}
\hline \multirow{2}{*}{$\begin{array}{c}\text { Dimensões das fibras } \\
(\mu \mathrm{m})\end{array}$} & \multicolumn{4}{c}{ Peneira (mesh) } \\
\cline { 2 - 5 } & 20 & 35 & 65 & 150 \\
\hline \multirow{2}{*}{ Comprimento } & $\begin{array}{c}90(1224-624) \\
(1,5)(0,2)\end{array}$ & $\begin{array}{c}956(1350-697) \\
(16,1)(1,7)\end{array}$ & $\begin{array}{c}996(1270-749) \\
(6,3)(0,6)\end{array}$ & $\begin{array}{c}868(1118-644) \\
(9,7)(1,1)\end{array}$ \\
& & & & \\
Largura & $20,5(25,9-16,5)$ & $20,6(27,4-14,7)$ & $21,6(26,9-15,3)$ & $21,0(25,1-15,1)$ \\
& $(0,92)(4,5)$ & $(0,7)(3,6)$ & $(0,6)(2,9)$ & $(0,3)(1,6)$ \\
Diâmetro do Lúme & $11,6(18,7-6,8)$ & $11,5(17,0-7,3)$ & $12,5(20,1-8,4)$ & $12,4(18,8-7,5)$ \\
& $(1,0)(8,9)$ & $(1,1)(9,1)$ & $(0,6)(4,5)$ & $(0,3)(2,1)$ \\
& & & & $4,5(7,3-1,2)$ \\
Espessura da Parede & $4,5(7,3-2,3)$ & $4,5(6,8-1,7)$ & $4,4(7,7-0,6)$ \\
& $(0,1)(2,0)$ & $(0,4)(7,7)$ & $(0,1)(1,3)$ & $(0,04)(0,9)$ \\
\hline
\end{tabular}

Valores médios seguidos de (máximo-mínimo) (desvio-padrão) e (coeficiente de variação).

Ci. Fl., v. 20, n. 3, jul.-set., 2010 
Determinação das propriedades físico-mecânicas dos painéis MDF

Os painéis MDF de eucalipto confeccionados em linha de produção industrial apresentaram, em relação aos produzidos em condições de laboratório, melhor desempenho para as propriedades de inchamento e absorção 24 horas, módulo de ruptura (MOR), elasticidade (MOE) e de resistência à tração superficial (Tabela 2).

Cabe destacar que, em linha de produção, os aditivos são aplicados à massa de fibras da madeira por meio de processo em linha de encolagem pressurizada ("blow line"), com a alta turbulência do sistema e aplicação atomizada dos aditivos promovendo um recobrimento eficiente da resina na superfície externa da parede das fibras. Em condições de escala laboratorial, os aditivos são aplicados por processo manual, utilizando pistolas de pressão, e que devem apresentar um recobrimento menos efetivo da parede externa das fibras.

Da mesma forma, cabe mencionar as diferenças quanto ao processo de prensagem dos colchões de fibras sendo que, em linha de produção, existe um gradiente decrescente de temperatura da cinta metálica entre as fases de entrada dos colchões de fibras e de saída dos painéis MDF. Em condições de laboratório, a temperatura utilizada na prensa em laboratório é fixada pela média da temperatura praticada na prensa industrial.

Considera-se que as maiores temperaturas existentes no início da prensagem dos colchões de fibras na prensa industrial devem promover uma cura mais eficiente da resina nas faces dos painéis, aumentando a sua dureza superficial e resultando em painéis MDF de maior resistência superficial (melhores propriedades de módulo de ruptura, módulo de elasticidade e resistência à tração superficial) e menores valores de inchamento e absorção 24 horas.

A literatura indica que há correlação positiva entre a densidade dos painéis e suas propriedades mecânicas (MALONEY, 1989), e que a densidade do painel MDF afeta positivamente os valores dos módulos de ruptura e de elasticidade (GANEV et al., 2005; CAI et al., 2006)

Os índices de correlação de Pearson entre as propriedades físico-mecânicas dos painéis MDF confeccionados em laboratório indicam uma

TABELA 2: Propriedades de painéis MDF obtidos em laboratório e linha de produção industrial.

TABLE 2: Properties of MDF panels manufactured in laboratory and industrial line production.

\begin{tabular}{|c|c|c|c|}
\hline Variáveis tecnológicas & Local & Valores & NBR 15316 \\
\hline $\begin{array}{l}\text { Densidade básica } \\
\left(\mathrm{kg} / \mathrm{m}^{3}\right)\end{array}$ & $\begin{array}{c}\text { Laboratório } \\
\text { Linha de Produção }\end{array}$ & $\begin{array}{l}695 \mathrm{a} \\
704 \mathrm{a}\end{array}$ & --- \\
\hline $\begin{array}{l}\text { Módulo de ruptura } \\
\left(\mathrm{N} / \mathrm{mm}^{2}\right)\end{array}$ & $\begin{array}{c}\text { Laboratório } \\
\text { Linha de Produção }\end{array}$ & $\begin{array}{l}36,1 \mathrm{a} \\
44,7 \mathrm{~b}\end{array}$ & Mín 20 N/mm² \\
\hline $\begin{array}{l}\text { Módulo de elasticidade } \\
\left(\mathrm{N} / \mathrm{mm}^{2}\right)\end{array}$ & $\begin{array}{c}\text { Laboratório } \\
\text { Linha de Produção }\end{array}$ & $\begin{array}{l}3776 \mathrm{a} \\
4283 \mathrm{~b}\end{array}$ & Mín 2200 N/mm² \\
\hline $\begin{array}{l}\text { Resistência à tração } \\
\text { perpendicular }\left(\mathrm{N} / \mathrm{mm}^{2}\right)\end{array}$ & $\begin{array}{c}\text { Laboratório } \\
\text { Linha de Produção }\end{array}$ & $\begin{array}{l}1,01 \mathrm{a} \\
1,00 \mathrm{a}\end{array}$ & Mín $0,55 \mathrm{~N} / \mathrm{mm}^{2}$ \\
\hline $\begin{array}{l}\text { Resistência à tração } \\
\text { superficial }\left(\mathrm{N} / \mathrm{mm}^{2}\right)\end{array}$ & $\begin{array}{c}\text { Laboratório } \\
\text { Linha de Produção }\end{array}$ & $\begin{array}{l}2,11 \mathrm{a} \\
2,40 \mathrm{~b}\end{array}$ & --- \\
\hline $\begin{array}{l}\text { Resistência arrancamento } \\
\text { parafuso topo (kgf) }\end{array}$ & $\begin{array}{c}\text { Laboratório } \\
\text { Linha de Produção }\end{array}$ & $\begin{array}{l}136 \mathrm{a} \\
135 \mathrm{a}\end{array}$ & --- \\
\hline $\begin{array}{l}\text { Resistência arrancamento } \\
\text { parafuso face (kgf) }\end{array}$ & $\begin{array}{c}\text { Laboratório } \\
\text { Linha de Produção }\end{array}$ & $\begin{array}{l}123 \mathrm{a} \\
126 \mathrm{a}\end{array}$ & --- \\
\hline $\begin{array}{l}\text { Inchamento em espessura } \\
(\%)\end{array}$ & $\begin{array}{c}\text { Laboratório } \\
\text { Linha de Produção }\end{array}$ & $\begin{array}{l}9,5 \mathrm{a} \\
5,8 \mathrm{~b}\end{array}$ & Máx $12 \%$ \\
\hline $\begin{array}{l}\text { Absorção de água } \\
(\%)\end{array}$ & $\begin{array}{c}\text { Laboratório } \\
\text { Linha de Produção }\end{array}$ & $\begin{array}{l}56,1 \mathrm{a} \\
33,7 \mathrm{~b}\end{array}$ & --- \\
\hline
\end{tabular}

Em que: Letras representam a comparação entre diferentes locais, para a mesma propriedade, ao nível de $5 \%$ de probabilidade (Teste de Tukey) - SAS. 
correlação estatisticamente significativa entre (i) os módulos de ruptura e de elasticidade, (ii) a densidade média e a resistência à tração perpendicular e (iii) o inchamento e absorção. Para os painéis MDF confeccionados em linha de produção industrial essas correlações são, da mesma forma, mantidas como tendência sem apresentar, no entanto, significância estatística (Tabela 3).

TABELA 3: Correlações entre as propriedades dos painéis MDF obtidos em laboratório e em linha de produção industrial.

TABLE 3: Correlation between the properties of MDF panels manufactured in laboratory and industrial line production.

\begin{tabular}{|c|c|c|c|c|c|c|c|c|c|}
\hline LABORATÓRIO & 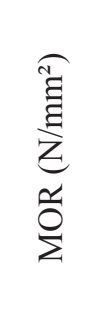 & 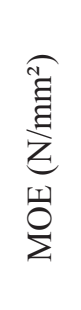 & 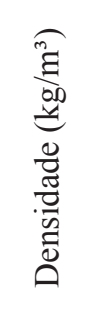 & 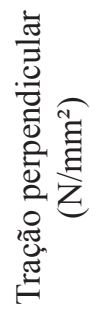 & 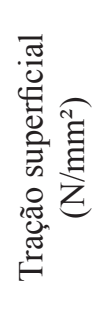 & 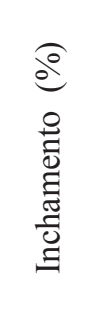 & 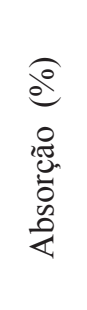 & 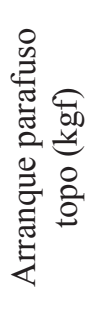 & 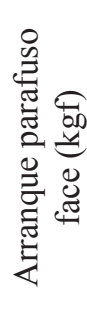 \\
\hline $\operatorname{MOR}\left(\mathrm{N} / \mathrm{mm}^{2}\right)$ & - & & & & & & & & \\
\hline $\operatorname{MOE}\left(\mathrm{N} / \mathrm{mm}^{2}\right)$ & $0,81 *$ & - & & & & & & & \\
\hline Densidade $\left(\mathrm{kg} / \mathrm{m}^{3}\right)$ & 0,01 & 0,01 & - & & & & & & \\
\hline Tração perpendicular $\left(\mathrm{N} / \mathrm{mm}^{2}\right)$ & 0,05 & 0,11 & $0,64 *$ & - & & & & & \\
\hline $\begin{array}{l}\text { Tração superficial } \\
\left(\mathrm{N} / \mathrm{mm}^{2}\right)\end{array}$ & 0,12 & 0,19 & 0,00 & $-0,22$ & - & & & & \\
\hline Inchamento $(\%)$ & $-0,03$ & 0,01 & $-0,26$ & 0,21 & $-0,33$ & - & & & \\
\hline Absorção (\%) & 0,14 & 0,21 & $-0,26$ & 0,17 & $-0,20$ & $0,90 *$ & - & & \\
\hline Arranque parafuso topo (kgf) & $-0,13$ & 0,00 & 0,17 & $-0,02$ & 0,15 & 0,22 & 0,27 & - & \\
\hline Arranque parafuso face (kgf) & 0,48 & 0,54 & 0,01 & $-0,13$ & 0,25 & 0,09 & 0,28 & 0,28 & - \\
\hline
\end{tabular}

Em que: *Valores que correspondem a correlações estatisticamente significativas a $p<0,05$.

LINHA DE PRODUÇÃO
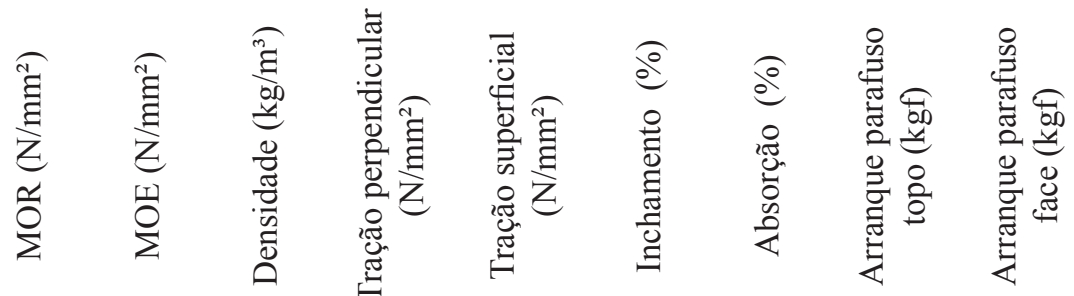

\begin{tabular}{|c|c|c|c|c|c|c|c|c|c|}
\hline $\operatorname{MOR}\left(\mathrm{N} / \mathrm{mm}^{2}\right)$ & - & & & & & & & & \\
\hline $\operatorname{MOE}\left(\mathrm{N} / \mathrm{mm}^{2}\right)$ & 0,63 & - & & & & & & & \\
\hline Densidade $\left(\mathrm{kg} / \mathrm{m}^{3}\right)$ & 0,12 & 0,11 & - & & & & & & \\
\hline Tração perpendicular $\left(\mathrm{N} / \mathrm{mm}^{2}\right)$ & $-0,11$ & $-0,01$ & 0,00 & - & & & & & \\
\hline Tração superficial $\left(\mathrm{N} / \mathrm{mm}^{2}\right)$ & 0,16 & 0,12 & 0,33 & 0,19 & - & & & & \\
\hline Inchamento $(\%)$ & 0,36 & 0,44 & $-0,2$ & $-0,36$ & 0,07 & - & & & \\
\hline Absorção (\%) & $-0,07$ & $-0,22$ & $-0,44$ & $-0,31$ & 0,09 & 0,50 & - & & \\
\hline Arranque parafuso topo (kgf) & $-0,05$ & $-0,51$ & $-0,01$ & $-0,08$ & $-0,03$ & $-0,30$ & 0,17 & - & \\
\hline Arranque parafuso face (kgf) & $-0,19$ & $-0,42$ & $-0,13$ & $-0,14$ & $-0,02$ & $-0,08$ & 0,35 & 0,31 & - \\
\hline
\end{tabular}

Em que: *Valores que correspondem a correlações estatisticamente significativas a $p<0,05$.

Ci. Fl., v. 20, n. 3, jul.-set., 2010 
Avaliação tecnológica de painéis MDF de madeira de Eucalyptus grandis confeccionados em ... 499

\section{CONCLUSÕES}

Os resultados do presente trabalho permitem concluir que (i) as fibras da madeira de eucalipto, após o processo de desfibramento, apresentaram rompimento das paredes no sentido transversal; (ii) os painéis MDF confeccionados com fibras de Eucalyptus grandis e obtidos em laboratório, em comparação aos de linha industrial, apresentaram menores valores médio de módulo de ruptura, de elasticidade e de resistência à tração superficial e pior desempenho para inchamento e absorção 24 horas; (iii) houve correlação estatisticamente significativa entre módulo de ruptura e elasticidade; densidade média e resistência à tração perpendicular e inchamento e absorção $24 \mathrm{~h}$ para os painéis MDF de laboratório; (iv) as mesmas tendências das correlações para os painéis MDF de linha de produção sem apresentar significância estatística; (v) os parâmetros de qualidade dos painéis MDF de laboratório e de fabricação industrial possibilitam monitorar as variáveis do processo e analisar as propriedades de chapas.

\section{AGRADECIMENTOS}

À Duratex S.Ae ao Laboratório de Anatomia e Identificação de Madeiras do Departamento de Ciências Florestais da ESALQ/USP.

\section{REFERÊNCIAS BIBLIOGRÁFICAS}

ANDRADE, A. M., et al. Efeitos da fertilização e da calagem do solo na produção e qualidade da madeira de eucalipto. Revista Árvore, Viçosa, v. 18, n. 1, p. 69-79, mai/jun. 1994.

ASSOCIAÇÃO BRASILEIRA DE NORMAS TECNICAS (NBR 15316). Chapas de fibra de média densidade. Parte 1 - Terminologia. Rio de Janeiro: ABNT, 2006. 4 p.

ASSOCIAÇÃO BRASILEIRA NORMAS TECNICAS (NBR 15316). Chapas de fibra de média densidade. Parte 3 - Métodos de ensaios. Rio de Janeiro: ABNT, 2006. 48 p.

BELINI, U. L. Caracterização e alterações na estrutura anatômica da madeira do Eucalyptus grandis em três condições de desfibramento e efeito nas propriedades tecnológicas de painéis MDF. 2007. 90 f. Dissertação (Mestrado em Tecnologia de Produtos Florestais) - Escola Superior de Agricultura “Luiz de Queiroz", Piracicaba, 2007. BELINI, U. L et al. Alterações na estrutura anatômica da madeira de cavacos de Eucalyptus grandis em três condições de desfibramento para a confecção de painéis MDF. Revista Árvore, Viçosa, v. 32, n. 3, p. 523 - 5324, maio/jun. 2008a.

BELINI, U. L et al. Efeito do desfibramento da madeira Eucalyptus grandis na morfologia das fibras e propriedades de painéis MDF. Revista Cerne, Lavras, v. 14, n. 3, p. 227 - 234, jul/set 2008 b.

BENADUCE, C. Fabricação de painéis de média densidade (MDF) a partir de fibras de Eucalyptus grandis e Pinus caribaea var. hondurensis. 1998. 113 f. Dissertação (Mestrado em Ciências e Tecnologia de Madeiras) - Escola Superior de Agricultura "Luiz de Queiroz", Piracicaba,, 1998.

BANCO NACIONAL DE DESENVOLVIMENTO ECONÔMICO E SOCIAL (BNDES). Área de Setores Produtivos 1. Painéis de Madeira Reconstituída. Brasília, 2002, 22 p.

CAMPOS, C. I. Produção e caracterização físico mecânica de MDF a partir de fibras de madeira de reflorestamento e adesivos alternativos em diversos teores. 2005, $127 \mathrm{f}$. Tese (Doutorado em Engenharia e Ciência dos Materiais) - EESC, 2005. CAI, Z; MUEHL, J. H.; WINANDY, J. Effects of panel density and mat moisture content on processing medium density fiberboard. Forest Products Journal. Madison, v. 56, n. 10, p. $20-$ 25, Oct. 2006.

ECKBLAD, J. W. How many samples should be taken. Bio Science, v. 41, p. 346-348. June 1991.

GANEV, S. et al. Mechanical properties of MDF as a function of density and moisture content. Wood and Fiber Science. v. 37, n. 2, p. 314 - 326, 2005. GORRINI, B. et al. Tableros de partículas y MDF de Eucalyptus nitens: ensayos en escala industrial. Bosque. Valdívia, v. 25, n. 3, p. 89-97, 2004

INTERNATIONAL ASSOCIATION OF WOOD ANATOMY (IAWA). List of microscopic features for wood identification. IAWA Bulletin, Leiden. v. 10, n. 3, p. 226-332, 1989.

IWAKIRI, S. et al. Painéis de madeira reconstituída. Curitiba: FUPEF, 2005. 247 p.

JOHANSEN, D. A. Plant microtechnique. New York: MacGraw-Hill. 1940. 533 p.

KRZYSIK, A. M. et al. Medium density fiberboards from plantation grown Eucalyptus saligna. Forest Products Journal. Madison, v. 51, n. 10, p. 47 - 50, 2001

MALONEY, T. M. Modern Particleboard \& dry process fiberboard manufacturing. San Francisco: Miller Freeman, 1989, 672 p.

ROCHA, F. T.; FLORSHEIM,S. M. B.; COUTO, 
H. T. Z. Variação das dimensões dos elementos anatômicos da madeira de árvores de Eucalyptus grandis aos sete anos. Revista do Instituto Florestal, São Paulo, v. 16, n. 1, p. 43-55. jun. 2004. SILVA, J. R. M. et al. Influência da morfologia das fibras na usinabilidade da madeira de Eucalyptus grandis. Revista Árvore, Viçosa, v. 29, n. 3, p. 479 - 487, maio/jun. 2005.

TECHNICAL ASSOCIATION OF THE PULP
AND PAPER INDUSTRY. Fiber length of pulp by classification. Atlanta: TAPPI, 1975.

TOMAZELLO FILHO, M. Variação radial da densidade básica e da estrutura anatômica da madeira do Eucalyptus grandis e E. saligna. Revista IPEF. Piracicaba. n. 29, p. 37 - 45, 1985a.

TOMAZELLO FILHO, M. Estrutura anatômica da madeira de oito espécies de eucalipto no Brasil. Revista IPEF. Piracicaba. n. 29, p. 25 - 36, 1985 b.

Ci. Fl., v. 20, n. 3, jul.-set., 2010 